\title{
Automatic Lesion Detection in Breast DCE-MRI
}

\author{
Stefano Marrone ${ }^{1}$, Gabriele Piantadosi ${ }^{1}$, Roberta Fusco ${ }^{2}$, Antonella Petrillo ${ }^{2}$, \\ Mario Sansone ${ }^{1}$, and Carlo Sansone ${ }^{1}$ \\ 1 DIETI - University of Naples Federico II, Italy \\ \{ste.marrone.g, gabriele.piantadosi\}@gmail.com, \\ \{carlosan, msansone\}@unina.it \\ 2 Dept. of Diagnostic Imaging, National Cancer Institute \\ of Naples 'Pascale Foundation', Italy \\ roberta.fusco@unina.it, antonellapetrillo2@gmail.com
}

\begin{abstract}
Dynamic Contrast Enhanced-Magnetic Resonance Imaging (DCE-MRI) has demonstrated in recent years a great potential in screening of high-risk women for breast cancer, in staging newly diagnosed patients and in assessing therapy effects. The aim of this work is to propose an automated system for suspicious lesion detection in DCE-MRI to support radiologists during patient image analysis. The proposed method is based on a Support Vector Machine trained with dynamic features, extracted, after a suitable pre-processing of the image, from an area preselected by using a pixel-based approach. The performance were evaluated by using a leave-one-patient-out approach and compared to manual segmentation made up by an experienced radiologist. Our results were also compared to other automatic segmentation methodologies: the proposed method maximises the area of correctly detected lesions while minimizing the number of false alarms (with an accuracy of 98.70\%).
\end{abstract}

Keywords: DCE-MRI, ROI detection, dynamic features, SVM.

\section{Introduction}

In recent years Dynamic Contrast Enhanced-Magnetic Resonance Imaging (DCE-MRI) has gained popularity as an important complementary diagnostic methodology for early detection of breast cancer [1. It has demonstrated a great potential in screening of high-risk women, in staging newly diagnosed breast cancer patients and in assessing therapy effects 2 thanks to its minimal invasiveness and to the possibility to visualize $3 \mathrm{D}$ high resolution dynamic (functional) information not available with conventional RX imaging 345.

One of the major issues in developing computer aided systems (CAD) for breast DCE-MRI is to detect the suspicious region of interests (ROIs) as sensibly as possibile, while simultaneously minimising the number of false alarms. This task is made harder by the peculiarity of DCE-MRI breast examinations: breast movements due to inspiration, huge diversity of lesion types. Moreover, a feasible CAD should be as fast as possible to operate in a clinical environment.

A. Petrosino (Ed.): ICIAP 2013, Part II, LNCS 8157, pp. 359-368, 2013.

(C) Springer-Verlag Berlin Heidelberg 2013 
It is worth noting that, while several papers addressed so far the problem of automatically classifying breast lesions in DCE-MRI [5 20], the automatic detection of suspicious ROIs is still an open problem 67.

The aim of this paper is to propose an automated method for detection of suspicious region of interest on breast DCE-MRI data to support radiologists during patient image analysis. The proposed methodology is based on a support vector machine (SVM) classifier trained on dynamic features, whose performance have been improved by a preliminary image pre-processing and a pixel-based pre-selection phase.

The paper is organized as follows. In Section 2 we describe the characteristics of recruited patients, the breast DCE-MRI data acquisition protocol, the experienced radiologist manual segmentation procedure, and the proposed automatic suspicious region segmentation algorithm. The obtained results are presented and discussed in Section 3, while in Section 4 we draw some conclusions.

\section{Materials and Methods}

\subsection{Patients}

The dataset is constituted of 26 women breast DCE-MRI 3D data, (average age 41 years, in range 26-69) with benign or malignant lesions histopathologically proven: 15 lesions were malignant and 11 were benign.

\subsection{Data Acquisition}

All patients underwent imaging with a 1,5T scanner (Magnetom Symphony, Siemens Medical System, Erlangen, Germany) equipped with breast coil. DCE T1-weighted FLASH 3D coronal images were acquired (TR/TE: 9,8/4,76 ms; flip angle: 25 degrees; field of view $330 \times 247 \mathrm{~mm}$ x mm; matrix: 256 x 128; thickness: $2 \mathrm{~mm}$; gap: 0; acquisition time: $56 \mathrm{~s}$; 80 slices spanning entire breast volume). One series $\left(t_{0}\right)$ was acquired before and 9 series $\left(t_{1}-t_{9}\right)$ after intravenous injection of $0.1 \mathrm{mmol} / \mathrm{kg}$ of a positive paramagnetic contrast agent (Gd-DOTA, Dotarem, Guerbet, Roissy CdG Cedex, France). An automatic injection system was used (Spectris Solaris EP MR, MEDRAD, Inc.,Indianola, PA) and injection flow rate was set to $2 \mathrm{ml} / \mathrm{s}$ followed by a flush of $10 \mathrm{ml}$ saline solution at the same rate.

\subsection{Manual Segmentation}

An experienced radiologist delineated suspect regions of interest (ROIs) using T1-weighted and subtractive image series. Starting from DCE acquired data, the subtractive image series is defined by subtracting $t_{0}$ series from $t_{4}$ series. In subtractive images any tissue that does not absorb contrast agent is suppressed. Manual segmentation stage was performed in Osirix 10, that allows user to define ROIs at a sub-pixel level. 


\subsection{Proposed Method}

The whole process can be modelled as a series of successive refinements (fig. 1) on patient DCE-MRI images, each one intended to highlight suspect lesions while eliminating noise, offsets and foreign tissues (muscle, bones, etc.). The result is a binary voxel by voxel labeling (intended for suspicious/not suspicious) whose union represent a Region Of Interest (ROI) to be submitted to expert radiologist's advice.

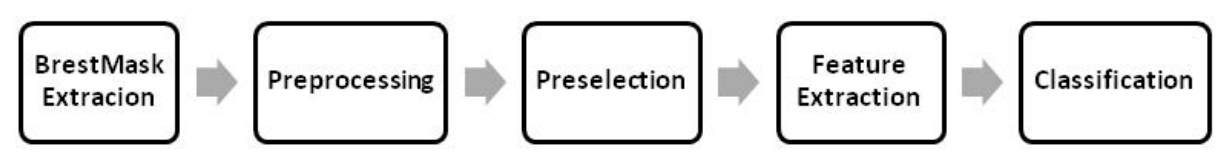

Fig. 1. Block diagram of the proposed method

Breast-mask Extraction. Breast-mask is a binary mask representing only breast parenchyma and excluding background and other tissues. The breastmask extraction represents a crucial stage in automated breast lesion segmentation algorithms to reduce the computational cost of feature extraction and attenuate noise caused by extraneous pixel.

Each slice contains background and tissue (breast parenchyma, organs, bones, etc.) pixels. In literature, several breast-mask segmentation algorithms have been proposed [89], some of them [114] based on Otsu thresholding [12].

Our breast-mask extraction procedure is divided into three main steps: a) preprocessing, b) Otsu thresholding, c) morphological refinements. The starting volume is the 3 -D data obtained as the minimum signal intensity $\left(S I_{m i n}\right)$, along time, of the original 4-D volume value. The pre-processing step use min-max normalisation in order to avoid sub-segmentation in slices that have small range of values. As last step, in order to smooth mask borders and fill internal holes, a cascade of morphological operators is applied in this order: Closing and Holes filling applied on global volume, Erosion applied slice by slice in coronal view direction, Dilatation applied on the global volume.

Preprocessing. Patient movements can affect the evaluation of dynamic MRI data. Often such motions involve a shift of one or two pixels. A pre-processing stage including image registration might improve the performance of tumor detection [13. A comprehensive way to deal with this issue would be non-rigid image registration. However, as non-rigid image registration involves cumbersome computation, often a faster rigid registration or no registration at all is used. In this study we adopted a median filter-based pre-processing: in this manner, small shifts are considered as noise superimposed on correctly aligned images. In section 3 we compared the performance of our approach to the case of rigid registration (by using the intensity-based image registration functions available in MatLab 2012a Image Processing Toolbox) or no registration at all. 
Pre-selection. Each voxel is associated with a Time Intensity Curve (TIC) representative of the temporal dynamics of the signal. The TIC reflects the absorption and the release of the contrast agent, following vascularisation characteristics of the tissue under analysis [14.

Moroever, for each time instant $k$ the Relative Enhancement (RE) is defined as eq. (1):

$$
R E(k)=\frac{T I C(k)-T I C(0)}{T I C(0)}
$$

RE curve still models the absorption of the contrast agent along time, but refers to it (to a first order approximation) in $\mathrm{mmol} / \mathrm{kg}$.

Pre-selection step is used to reduce the number of voxels to be submitted to the classifier: each voxel is pre-classified with the algorithm used in 15] except for the $t_{9}$ bond. All voxel in which $\max (R E(t))$ is below $30 \%$ of the basal signal are marked as not-suspicious while all the others are marked as unsure and will be submitted to the subsequent classification step.

Feature Extraction. Starting from data provided by DCE-MRI images is possible to extract several sets of feature, representative of specific characteristics and properties. Among them, it is worth mentioning Dynamic, Spatial-Temporal and Textural [16] features. In our work we used dynamic features that demonstrated to be the best choice in breast cancer automatic segmentation problem [16.

Dynamic features reflect information about the temporal dynamics of the signal, evaluated by direct measurements of the TIC for each voxel. They are defined on mathematical models (but not on pharmacokinetics) and this makes them computationally efficient and robust to contrast agent variation. Several features belonging to the dynamic category have been proposed so far in the literature. Among them, it is worth mentioning those presented in [174/5]. The dynamic features used in our work were:

- Area under TIC (AUC), total amount of contrast agent absorbed;

- Relative Enhancement at Maximum Point (ReMax), contrast agent peak;

- Time To Peak (TTP), the time in which ReMax is gained;

- Wash-In Slope (WIS), angular coefficient of linearized approximation of TIC curve from time 0 to TTP;

- Wash-Out Slope (WOS), angular coefficient of linearized approximation of TIC curve from time TPP to last time;

Classification. Most of our work has focused first on choosing the best classifier and then optimizing the parameters of the classifier itself. With best classifier we mean the one which best highlight potential suspect lesions, minimizing at same time false alarms. To deal with this, the dataset has been splitted in two parts, one composed of 4 patients (whose dynamic characteristics well represents the whole dataset) and one composed of the remaining 22 patients. The first one was used in the optimization phase; while the second one in experimental phase, in which a leave-one-patient-out approach was used to statistically validate results. 
The output of the classification step is the union of all voxel labeled as suspect that represent suspect regions which will be subject to expert radiologist advice.

The proposed methodology uses a Support Vector Machine (SVM) classifier (with third-degree Radial Basis Function kernel; unitary cost; eps $10^{-3}$ ). The SVM turned out to be the best when compared to a Multi-Layer Perceptron (MLP) (learning rate 0.3, momentum 0.2, training epoch 500) and to a Random Forest (RF) classifier (made up of 10 Random Trees each one using a random subset of feature with no limitation on its maximum depth).

We considered as suspicious voxels those contained in the ROI manually segmented by the experienced radiologist (A.P.). Not suspicious voxels were instead randomly chosen inside the pre-selection mask (but outside the suspicious ROI).

As outlined in Fig. 2, separating suspicious from pre-selection mask voxels is much more difficult than separating not-suspicious from pre-selection mask voxels, since the TICs of the first two kinds of voxels are more similar than those belonging to the second two kinds of voxels. As a matter of fact, we noticed that classifiers trained on suspicious and pre-selected voxels had a greater generalization ability with respect to ones trained on suspect and not suspect ones: in particular in the second case, the obtained classifier is unable to reliably recognize suspect voxel from not suspect ones, generating an output very close to the pre-selection mask.

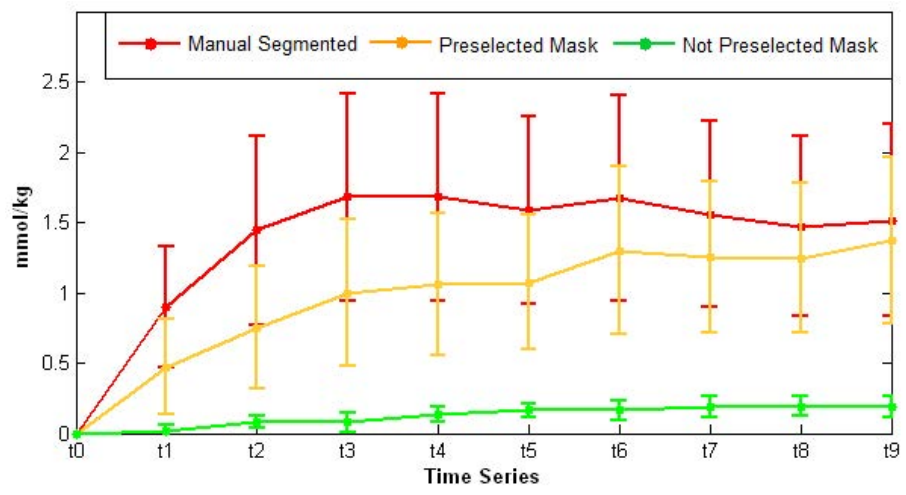

Fig. 2. Examples of average TICs over the three types of ROIs. Vertical bars represent standard deviations.

Finally, it is worth noticing that a random choice is necessary in order to minimize the possibility of feeding the classifier with suspicious voxel not highlighted by the radiologist (because they belong to a very small region or because we do not have any histological proof).

In section 3 all the results obtained by varying the classifier, the preprocessing and the pre-selection methods are reported, in terms of Accuracy, Sensitivity and Specificity [18]. 


\section{$3 \quad$ Experimental Results}

Figure 3 summarizes the results produced by each step of the proposed method for a benign lesion, while Figure 4 shows the results for a malignant lesions.
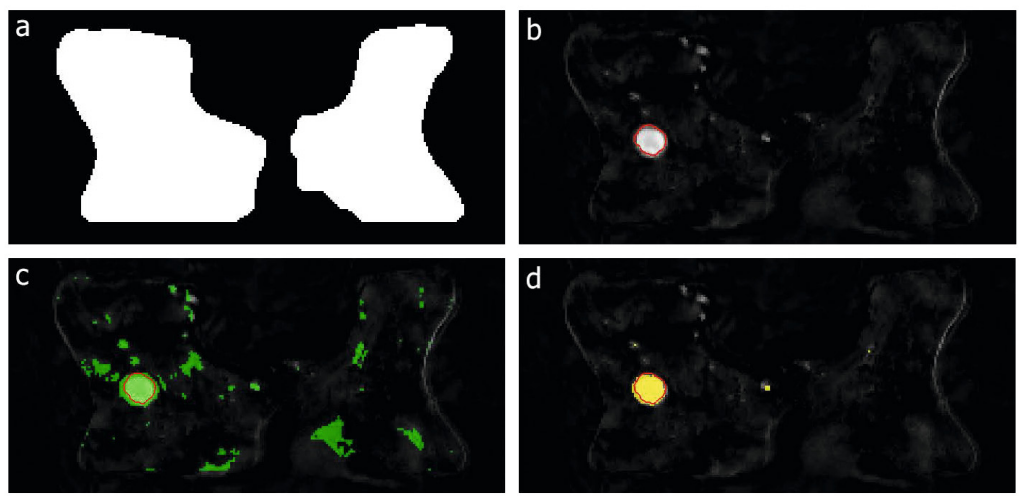

Fig. 3. A benign lesion: a) Brest-mask; b) Manual ROI lesion segmentation (red perimeter); c) Pre-selection mask (green area); d) automatic detected ROI (yellow area)
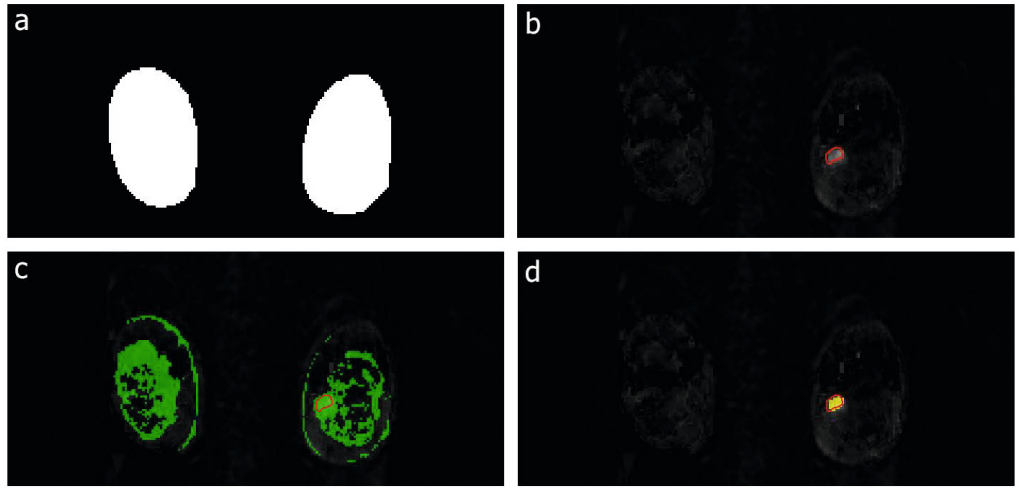

Fig. 4. A malignant lesion: a) Brest-mask; b) Manual ROI lesion segmentation (red perimeter); c) Pre-selection mask (green area); d) automatic detected ROI (yellow area)

Table 1 reports the results of the evaluation study with and without preprocessing and pre-selection phase, and by varying the classifer. For each combination the table reports the mean value (evaluated on a leave-one-patient-out basis) of sensitivity, specificity and accuracy, in decreasing order of accuracy. These results suggest that our approach with a SVM classification can give simultaneously a great accuracy and specificity, and a good value of sensitivity. 
It is also worth noticing that results in terms of sensitivity are even better in practice, since they refer to voxels and not to ROIs. Since the purpose of the proposed method is to support the radiologist in his work, the best method is in fact the one that maximises accuracy (in order to increase the probability to correctly recognize suspect regions) and specificity (in order to reduce false alarms), looking at the same time to an acceptable sensitivity (to be almost sure to recognize a satisfying ROI area).

Moreover, from Table 1 it can be observed that, by considering the accuracy, the results obtained by using the median registration outperform those obtainable with a rigid registration, which, in turn, are better with respect to those achieved without registration. The obtained results also confirmed that the use of a RE based voxel pre-selection gave always rise to better results with respect to the case when no preselection is used. When preselection was used, the SVM classifer perfomed consistently better than MLP and RF (differences in accuracy are always statistically significant $(p<0.05))$.

Table 1. ROI detection performance

\begin{tabular}{cccccc}
\hline PreProcessing PreSelelection & Classifier & Accuracy & Sensitivity & Specificity \\
\hline Median & Yes & SVM & $98.70 \%$ & $71.56 \%$ & $98.94 \%$ \\
Median & Yes & RF & $98.65 \%$ & $67.78 \%$ & $98.95 \%$ \\
Median & Yes & MLP & $98.63 \%$ & $72.82 \%$ & $98.85 \%$ \\
Rigid Reg. & Yes & SVM & $98.57 \%$ & $67.13 \%$ & $98.85 \%$ \\
Rigid Reg. & Yes & MLP & $98.48 \%$ & $68.79 \%$ & $98.74 \%$ \\
Rigid Reg. & Yes & RF & $98.40 \%$ & $64.19 \%$ & $98.70 \%$ \\
None & Yes & SVM & $98.39 \%$ & $69.82 \%$ & $98.66 \%$ \\
None & Yes & RF & $98.31 \%$ & $65.92 \%$ & $98.62 \%$ \\
None & Yes & MLP & $97.87 \%$ & $72.61 \%$ & $98.13 \%$ \\
Median & No & RF & $95.07 \%$ & $90.81 \%$ & $95.10 \%$ \\
Median & No & SVM & $94.75 \%$ & $92.74 \%$ & $94.75 \%$ \\
Rigid Reg. & No & RF & $94.28 \%$ & $90.19 \%$ & $94.30 \%$ \\
None & No & RF & $94.19 \%$ & $90.74 \%$ & $94.21 \%$ \\
Rigid Reg. & No & SVM & $94.00 \%$ & $91.20 \%$ & $94.00 \%$ \\
Rigid Reg. & No & MLP & $93.97 \%$ & $92.18 \%$ & $93.98 \%$ \\
None & No & MLP & $93.75 \%$ & $90.65 \%$ & $93.76 \%$ \\
Median & No & MLP & $93.73 \%$ & $92.82 \%$ & $93.71 \%$ \\
None & No & SVM & $93.61 \%$ & $91.08 \%$ & $93.61 \%$ \\
\hline
\end{tabular}

Table 2 reports our best result compared with other methodologies: a pixelbased approach proposed by Torricelli et al. [19], another approach based on dynamic features and MLP classifier [20, and a pixel-based approach based on $\mathrm{RE}$ (which is indeed our preselection methodology as described in \$2.4). As it is evident, our method demonstrated the best accuracy, with a sensitivity which is significantly higher than the second best. As regards the other two approaches, the difference among their accuracies and the one obtained by our approach is statistically signifcant $(p<0.05)$. 
Table 2. Performance comparison of the proposed method with other approaches

\begin{tabular}{|c|c|c|c|}
\hline Methodology & Accuracy & Sensitivity & Specificity \\
\hline Proposed Methodology & $98.70 \%$ & $71.56 \%$ & $98.94 \%$ \\
\hline Torricelli et al. 19. & $98.69 \%$ & $25.80 \%$ & $99.49 \%$ \\
\hline Fusco et al. 20] & $86.99 \%$ & $90.97 \%$ & $86.99 \%$ \\
\hline Pixel-Based on RE [subsec. 2.4 & $86.59 \%$ & $75.44 \%$ & $86.64 \%$ \\
\hline
\end{tabular}
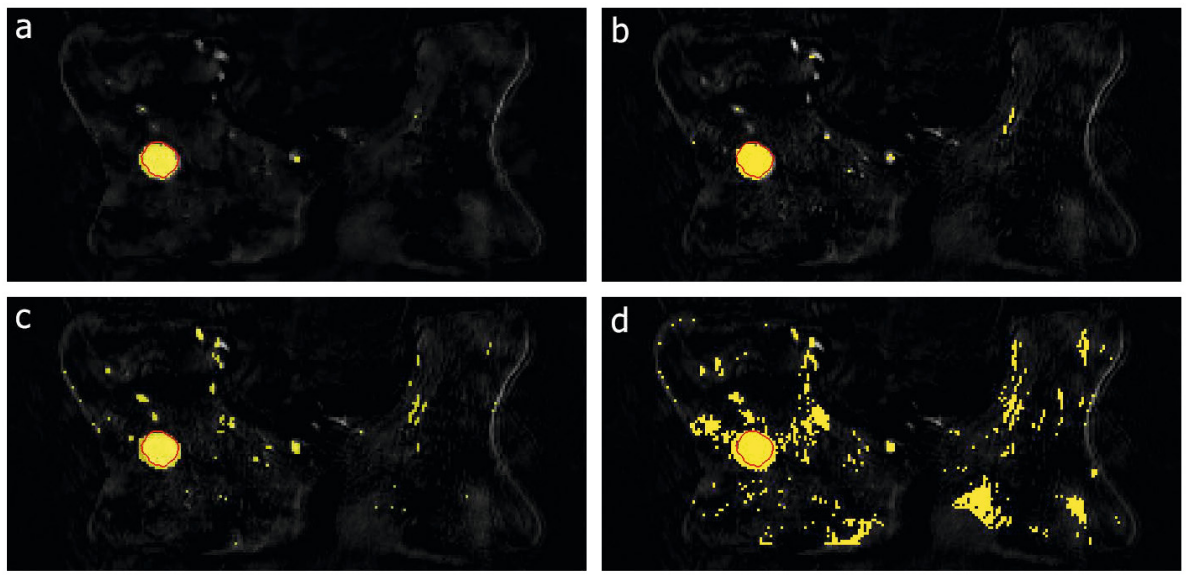

Fig. 5. Comparing results for a benign lesion: a) our proposed approach; b) the pixelbased approach proposed by Torricelli et al; c) the MLP-based approach proposed by Fusco et al; d) preselection mask
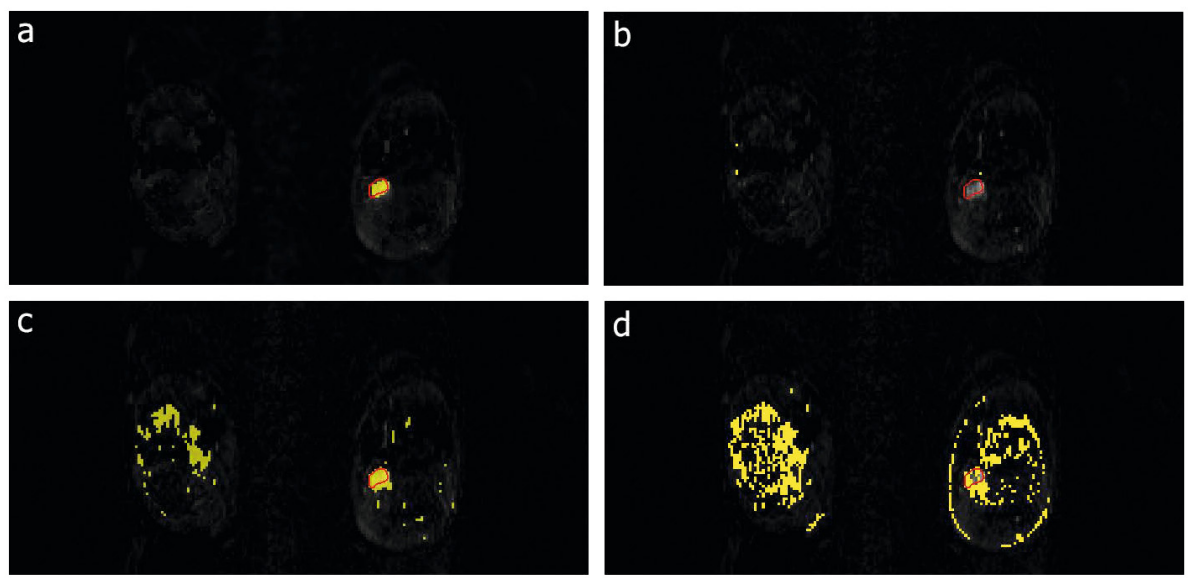

Fig. 6. Comparing results for a malignant lesion: a) our proposed approach; b) the pixel-based approach proposed by Torricelli et al; c) the MLP-based approach proposed by Fusco et al.; d) preselection mask. Note that in this case b) was not able to detect the lesion. 
In order to better analyze the results, figures 5 and 6 compare the automatic segmented region obtained for a benign and a malignant lesion, respectively, by using the approaches reported in table 2. In this case the advantage of using our approach appears even more evident.

\section{Conclusions and Future Works}

In this study we have proposed an automated system for suspicious lesion segmentation in breast DCE-MRI. The proposed method is based on a Support Vector Machine classifier trained on dynamic features extracted, after a suitable pre-processing of the image, from an area pre-selected by using a pixel-based approach.

The performance of the proposed method have been evaluated with respect to manual segmentation made up by an expert radiologist on a dataset of 26 breast lesions. Obtained results were very interesting and compared favourably with respect to other approaches.

Currently, the whole method is implemented in a Matlab tool that allows to analyze any patient image respecting the same protocol; we are also working on an Osirix plug-in.

It is worth noting that although the number of patients has grown over previous studies from 10 to 26 [15] is not yet possible to achieve a broader analysis because of the difficulties to enroll patients with the same data acquisition protocol and the absence of a public database.

We are planning to extend our study to a larger number of patients, to a larger kind of breast tumors, and to different examination protocols in order to extend the applicability of proposed system. Finally, our future work will focus on automatic malignant grade detection.

\section{References}

1. Lehman, C.D., et al.: MRI evaluation of the contralateral breast in women with recently diagnosed breast cancer. The New England Journal of Medicine 356, 1295-1303 (2007)

2. Olsen, O., et al.: Screening for breast cancer with mammography. Cochrane Database of Systematic Reviews CD001877 (2001)

3. El-Kwae, et al.: Detection of Suspected Malignant Patterns in Three-Dimensional Magnetic Resonance Breast Images. Journal of Digital Imaging (1998)

4. Twellmann, T., et al.: Image fusion for dynamic contrast enhanced magnetic resonance imaging. BioMedical Engineering OnLine (2004)

5. Levman, et al.: Classification of dynamic contrast-enhanced magnetic resonance breast lesion by support vector machines. IEEE Trans. Medical Imaging (2008)

6. Renz et al.: Detection and classification of contrast-enhancing masses by a fully automatic computer-assisted diagnosis system for breast MRI. J. Magn. Reson. Imaging (2012)

7. Vigniatti, et al.: Performance of a fully automatic lesion detection system for breast DCE-MRI. J. Magn. Reson. Imaging (2012) 
8. $\mathrm{Wu}$, et al.: Automated chest wall line detection for whole-breast segmentation in sagittal breast MR images. Med. Phys (2013)

9. Ortiz, et al.: Automatic atlas-based segmentation of the breast in MRI for 3D breast volume computation. Med. Phys (2012)

10. Rosset, A., et al.: OsiriX: An Open-Source Software for Navigating in Multidimensional DICOM Images. Journal of Digital Imaging 17(3), 205-216 (2004)

11. Twellmann, T., et al.: Detection of suspicious lesion in dynamic contrast enhanced MRI data. In: Annual International Conference of the IEEE Engineering in Medicine and Biology Society (2004)

12. Otsu, N., et al.: A threshold selection method from gray level histograms. IEEE Trans. Systems, Man and Cybernetics 9(1), 62-66 (1979)

13. Tanner, C., et al.: Does registration improve the performance of a computer aided diagnosis system for dynamic contrast-enhanced MR mammography? In: ISBI (2006)

14. Kuhl, C.K., et al.: Dynamic breast MR imaging: are signal intensity time course data useful for differential diagnosis of enhancing lesion? Radiology (1999)

15. Fusco, R., Sansone, M., Sansone, C., Petrillo, A.: Selection of Suspicious ROIs in Breast DCE-MRI. In: Maino, G., Foresti, G.L. (eds.) ICIAP 2011, Part I. LNCS, vol. 6978, pp. 48-57. Springer, Heidelberg (2011)

16. Fusco, R., et al.: Segmentation and classification of breast lesions using dynamic and textural features in Dynamic Contrast Enhanced-Magnetic Resonance Imaging. Computer-Based Medical Systems (CBMS) (2012)

17. Mayer-Baese, A., et al.: Small lesion evaluation based on unsupervised cluster analysis of signal-intensity time courses in dynamic breast MRI. International journal of Biomedical Imaging (2010)

18. Udupa, J.K., et al.: A framework for evaluating image segmentation algorithms. Computerized Medical Imaging and Graphics: The Official Journal of the Computerized Medical Imaging Society 30, 75-87 (2006)

19. Torricelli, P., et al.: Gadolinium-enhanced MRI with dynamic evaluation in diagnosing the local recurrence of rectal cancer. Abdom Imaging 28, 19-27 (2003)

20. Fusco, R., Sansone, M., Petrillo, A., Sansone, C.: A Multiple Classifier System for Classification of Breast Lesions Using Dynamic and Morphological Features in DCE-MRI. In: Gimel'farb, G., Hancock, E., Imiya, A., Kuijper, A., Kudo, M., Omachi, S., Windeatt, T., Yamada, K. (eds.) SSPR\&SPR 2012. LNCS, vol. 7626, pp. 684-692. Springer, Heidelberg (2012) 